\title{
In situ Atomic-scale Investigation of Elemental Diffusion in Pt-Co Nanoparticles
}

\author{
M. Chi, ${ }^{1}$ K.L. More, ${ }^{1}$ C. Wang, ${ }^{2}$ N. Markovic, ${ }^{2}$ and V. Stamenkovic ${ }^{2}$ \\ 1. Material Science \& Technology Division, Oak Ridge National Laboratory, Oak Ridge, TN, USA \\ 2. Department of Chemical \& Biomolecular Engineering, Johns Hopkins University, \\ Baltimore, MD, USA \\ ${ }^{3 .}$ Materials Science Division, Argonne National Laboratory, Argonne, IL, USA
}

Platinum alloyed with $3 d$ transition metals (TM), e.g. Fe, Co and $\mathrm{Ni}$, are strong candidate catalysts for the oxygen reduction reaction (ORR) in fuel cells [1,2]. Previous work on well-defined extended surfaces has demonstrated that ultrahigh ORR activity can be achieved for bi-metallic alloys with Ptsegregated surfaces, e.g., a Pt-skin layer and a Co-enriched subsurface layer in the case of $\mathrm{Pt}_{3} \mathrm{Co}$ [3]. The formation of uniform, well-defined, Ångström-scale surface layers (or shells) on each nanoparticle (NP), while keeping the NP diameters consistently less than $10 \mathrm{~nm}$, however, remains a considerable challenge. The most frequently used method to modify the elemental distribution at NP surfaces is thermal annealing and understanding the dynamics of the elemental diffusion within the NPs is critical to controlling the skin-layer structure and composition.

In this work, we studied the chemical distribution and atomic structure evolution of $\mathrm{Pt}_{3} \mathrm{Co}$ catalyst NPs during in situ thermal annealing by combining atomic-resolution Z-contrast imaging and energy dispersive spectroscopy (EDS) elemental mapping. A JEOL 2200FS microscope equipped with a CEOS Cs aberration corrector and a Bruker X-flash Si-drift detector (SDD) was used. TEM specimens were prepared by placing a drop of carbon-supported nanoparticles which were dispersed in a methonal solution onto Protochips Aduro ${ }^{\mathrm{TM}}$ thermal devices. A Protochips heating holder was used for the in situ heating experiments to increase the temperature (at a rate of $10^{6}{ }^{\circ} \mathrm{C} / \mathrm{sec}$ [4]) from $200^{\circ} \mathrm{C}$ to $600^{\circ} \mathrm{C}$ in $50^{\circ} \mathrm{C}$ steps with a 60 min hold at each temperature step.

The starting NPs were revealed to be homogeneous Pt-Co alloys based on both Z-contrast imaging and EDS elemental mapping. No obvious $\mathrm{Pt}_{3} \mathrm{Co} \mathrm{NP}$ sintering was observed during in situ annealing until a temperature of $550^{\circ} \mathrm{C}$ was reached, which was mostly a result of the uniformity of both the initial particle size and dispersion. We monitored 50 individual NPs during the annealing experiments to track structural and compositional changes. The formation of a Pt-rich shell was observed for most of the NPs when the temperature reached $450^{\circ} \mathrm{C}$ after a 30 min hold; however, a small number $(\sim 35 \%)$ of the $\mathrm{Pt}_{3} \mathrm{Co}$ NPs never formed a Pt-skin layer, even when the temperature was increased to $650^{\circ} \mathrm{C}$. This result suggests that simple annealing cannot be used to uniformly configure a Pt-skin layer structure on individual $\mathrm{Pt}_{3} \mathrm{Co} \mathrm{NPs}$, which also agrees with electrochemical characterization data showing that thermally annealed $\mathrm{Pt}_{3} \mathrm{Co}$ catalysts did not exhibit a catalytic activity as high as expected. When the same NPs were further annealed from $450^{\circ} \mathrm{C}$ to $600^{\circ} \mathrm{C}$, structural ordering of the $\{110\}$ planes was observed. First-principle calculations were used to investigate the observed inhomogeneities of Pt-skinlayer formation amongst $\mathrm{Pt}_{3} \mathrm{Co} \mathrm{NPs}$ [5]. It was found that $\mathrm{Pt}_{3} \mathrm{Co}$ alloy $\mathrm{NPs}$ having perfect cubeoctahedral shapes formed Pt-rich skin layer structures after 2 million sequential Monte Carlo simulations at $400^{\circ} \mathrm{C}$. The Pt concentrations in the resultant NPs are 99 at.\% in the outermost surface skin layer, 44 at. $\%$ in the second sub-surface layer, and 92 at.\% in the third sub-surface layer. On the contrary, for a 
particle of the same shape and size, but with an disordered outermost surface layer, the simulation showed Pt concentrations of 70 at.\% in the outermost surface layer, 80 at.\% in the second sub-surface layer, and 71 at.\% in the third sub-surface layer. These results demonstrate that elemental diffusion in $\mathrm{Pt}_{3} \mathrm{Co}$ NPs is extremely sensitive to the surface atomic structure of the as-synthesized NPs. Surface defects, such as vacancies or impurities, play a critical role in preventing the formation of a Pt-rich skin layer. Unfortunately, synthesizing a "perfect" surface structure on every NP is experimentally impossible. A more controlled method was investigated to form Pt-rich skin layers: acid leaching followed by thermal annealing. In this process, the acid leaching step results in a Pt-skeleton surface structure (the TM is leached out preferentially) and the annealing step relaxes the surface structure such that the surface recrystallizes at the atomic scale. In situ and ex situ microscopy of acid-leached $\mathrm{Pt}_{3} \mathrm{Co}$ NPs validated this concept (Fig. 1). Significant improvement in catalytic activity was observed for preleached $\mathrm{Pt}_{3} \mathrm{Co}$ NPs compared to those prepared by simple annealing [6]. Our results demonstrate the importance of investigating the atomic structure and composition of individual NPs, and they provide guidance for tailoring the properties of nanomaterials through surface modification [7].

References:

[1] T Toda, et al., J. Electrochem. Soc. 146[10], (1999) p. 3750.

[2] VR Stamenkovic, et al., Nature Materials 6[3], (2007) p. 241.

[3] VR Stamenkovic,. et al., Science 315, (2007) p.493.

[4] L.F. Allard, et al., Microscopy Research \& Technique 72(3), (2003), p. 208.

[5] C Wang, et al., J. Phys. Chem. C 45[113], (2009), p. 19365.

[6] C Wang, et al., J. Am. Chem. Soc. 36[133], (2011), p.14396.

[7] Research supported by the Fuel Cell Technology Program, Office of Energy Efficiency and

Renewable Energy, US Department of Energy (DOE) and by ORNL's ShaRE User Facility, which is sponsored by the Office of Basic Energy Sciences, U.S. DOE.

(a)

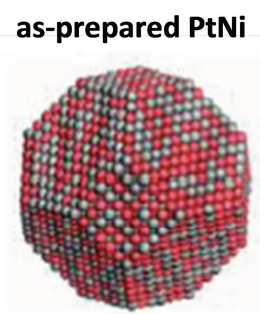

(b)

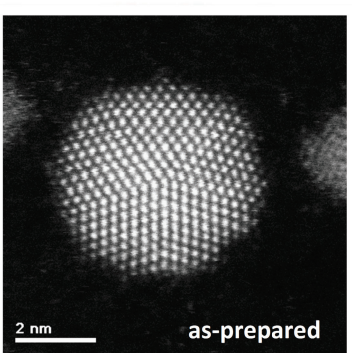

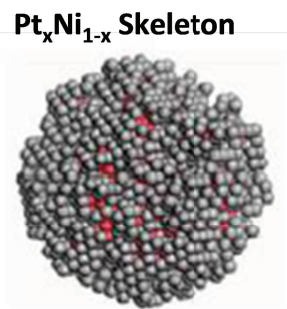

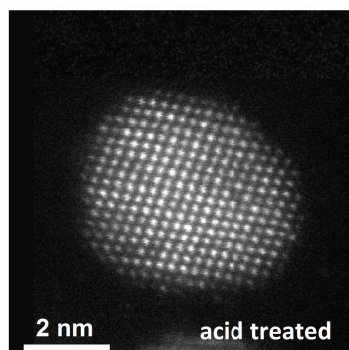

PtNi/Pt skin
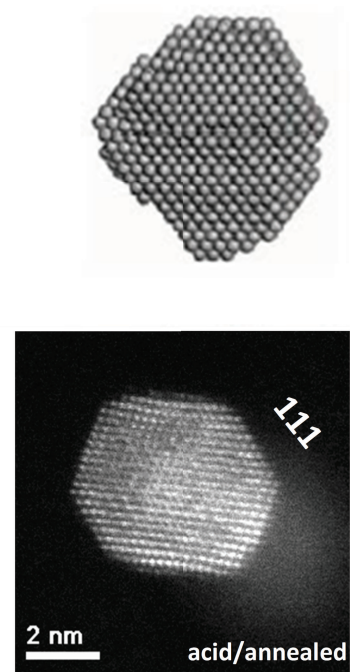

Figure 1. Atomic structure evolution of $\mathrm{Pt}_{3} \mathrm{Ni} \mathrm{NPs}$ upon annealing with prior acid-leaching. In situ TEM heating experiments reveal the formation of a Pt-rich skin layer. ppropriate. 